\title{
EDUCACIÓN SUPERIOR IBEROAMERICANA: UN ANÁLISIS MÁS ALLÁ DE LAS PERSPECTIVAS MERCADOLÓGICAS DE LA PRODUCCIÓN DE CONOCIMIENTO ${ }^{1}$
}

\author{
ALMEIDA, M. de L. P. de; CATANI, A. M. Educação superior iberoamericana: \\ uma análise para além das perspectivas mercadológicas da produção de \\ conhecimento. Buenos Aires: CLACSO, 2015.
}

En esta reseña se presenta una mirada preliminar de la obra organizada por Maria de Lourdes Pinto de Almeida y Afrânio Mendes Catani denominada Educação superior iberoamericana: Uma análise para além das perspectivas mercadológicas da produção de conhecimento, publicada en 2015 por la editora de la Clacso. Se trata de una recopilación de artículos de autores brasileños y extranjeros que se dedican a la temática de la Educación Superior y su importancia para el desarrollo de la Educación de los países iberoamericanos, dando especial énfasis al papel social de la universidad pública y privada y de la investigación ante las demandas del mercado globalizado y de las contradicciones y limitaciones del capitalismo. La obra está organizada en tres partes: la primera, nombrada Políticas educacionais no contexto da Educação Superior cuenta con cuatro capítulos; la segunda, Estudos de caso em Argentina, Chile e Brasil presenta siete capítulos; y la tercera, denominada Perspectivas mercadológicas na produção de conhecimento tiene tres capítulos en su estructura.

El primer capítulo, Las políticas educativas en Latinoamérica: 1990-2012. Derroteros: avances y preocupaciones, escrito por Cesar Gerónimo Tello, desarrolla una reflexión acerca de las principales políticas educacionales en América Latina de los últimos veinte años, buscando desvelar sus avances y limitaciones, describiendo las características de este recorte temporal y trayendo una discusión acerca de los conceptos de neoliberalismo y pos-neoliberalismo en los gobiernos latinoamericanos. Para el autor, esta delimitación de periodo histórico trae como características la presión de los organismos internacionales, el surgimiento de nuevos perfiles de Estado, cambios de gobiernos con continuidades y no continuidades en las políticas educacionales, llevándolo a concluir que ha habido pocos cambios significativos en 
estos veinte últimos años, a pesar de que se presenta como un periodo con dos modelos diferenciados de gestión de políticas sociales transitando en el escenario político.

En el segundo capítulo, Docencia e Investigación en el contexto de la Universidad actual, Antonio Bolívar y Rosel Bolívar Ruano, de España, traen como aspecto principal una discusión rica y actual sobre la identidad y las tareas del trabajo académico en la investigación y en la docencia, inicialmente por medio de una reflexión acerca de la génesis y del papel de la Universidad y de la docencia en términos de conceptualización. Además, ubican a la docencia y a la investigación en el espacio pedagógico de manera a establecer un equilibrio entre las relaciones de interés de la docencia y de la investigación. Para los autores, es necesario situar a la educación dentro del trabajo académico en el mismo nivel que la investigación, siguiendo a los conceptos desarrollados por Boyer (1990) y Shulman (2004) sobre la scholarship. Según ellos, una educación entendida como investigación recae en la necesidad de investigarse a la educación para alcanzar la requerida calidad en la docencia, siendo que la educación superior debe ser desarrollada juntamente con la investigación en un ambiente académico.

Con el intuito de reflexionar sobre la responsabilidad social e la universidad en sus propósitos históricos básicos y sobre el compromiso/alienación de los actores del ambiente académico, José Camilo dos Santos Filho es el autor del tercer capítulo denominado Propósitos da universidade e novas formas de sua responsabilidade social. En él, el autor discute la esencia e histórico de la universidad y su papel social, y considerando la visión de los activistas, de los defensores de la multiversidad y de los conservadores, propone nuevas alternativas para la responsabilidad social de la universidad, con base en la concepción de scholarship de la docencia universitaria.

En el cuarto capítulo, Ações afirmativas e política de cotas no Brasil: posições político-sociais em debate, los autores Sandra Veit Pillatti y Elton Luiz Nardi abordan las nuevas configuraciones de las acciones afirmativas y de la acción del Estado en este campo, enfatizando el sentido del sistema de cuotas y el valor atribuido a la reserva de vacantes como forma de ingreso en la universidad pública. Ellos abordan el proceso histórico y naturaleza de las acciones afirmativas, desde el surgimiento del término a nivel internacional hasta la configuración del sistema de cuotas sociales y raciales como acceso a la educación superior pública, dando énfasis a la forma como el tema ha sido abordado en distintas áreas. Teniendo como enfoque las acciones afirmativas como política pública abordadas a partir de diferentes estudios, los autores evidencian disonancias en su papel como "atenuador" de las desigualdades en el país y discuten el término igualdad a la luz de una perspectiva socio jurídica. 
El capítulo que abre el segmento de la obra sobre los estudios de caso en Argentina, Chile y Brasil es de autoría de Carlos Francisco Mazzola, titulado La Universidad de los gobiernos Kirchner. Mazzola analiza a la universidad pública en los tres últimos gobiernos de Argentina, evidenciando la forma de conducción "familiar" del tema en el país. Analiza datos estadísticos (matrículas, áreas, crecimiento de las ciencias sociales), históricos, marcos legales, formas de financiamiento y políticas, las continuidades y cambios en las Universidades desde los años 90. Él concluye que, en el periodo analizado, hay un grupo que defiende avances en el área, así como un grupo que está en la lucha por modificaciones en este sistema.

Con respecto a la Educación Superior en Chile, María Verónica Leiva Guerrero y María Leonor Conejeros Solar desarrollaron el capítulo titulado El cambio en los sistemas de financiamiento de la educación superior chilena: ¿lucro o calidad?. Las autoras analizan las políticas de financiamiento de la educación superior en Chile bajo la óptica chilena de valoración de la educación superior de la universidad, a partir de una perspectiva histórica. Destaca las alteraciones ocurridas en la acción del Estado entre el periodo de la dictadura y retorno de la democracia. En este periodo, se crearon nuevas directrices para regular la participación de las instituciones privadas. Presentan datos estadísticos sobre el crecimiento de universidades públicas y privadas y los marcos legales del proceso. Concluyen que hubo muchos cambios, principalmente el incremento de la demanda, el fortalecimiento de las universidades privadas con el aumento del financiamiento público, perjudicando la lógica de la equidad.

En el capítulo denominado Políticas de educação superior no Brasil: um estudo da relação da Universidade Estadual de Campinas (UNICAMP) com o setor produtivo (1980-1992), Maria de Lourdes Pinto de Almeida analiza históricamente la trayectoria de la Unicamp desde 1980 hasta la gestión de Carlos Vogt a partir de una perspectiva socio política. Para la autora, la integración entre la universidad pública y las empresas privadas fue marcada por las inestabilidades económicas y políticas del país, teniendo influencias financieras en la realización de investigaciones académicas y de enseñanza, con demandas provenidas de las empresas, evidenciando el fondo lucrativo de la enseñanza y de la investigación públicos, además de influenciar en los rumbos administrativos de estas instituciones públicas. Para comprender esta inclinación de la institución pública para recorrer al recurso privado, es necesario comprender la transformación del papel del conocimiento, del intelectual y del Estado, y asimismo las tendencias hegemónicas vigentes, sin olvidarnos de las consecuencias de los "incentivos" públicos para la privatización de la investigación. 
Mirian Lúcia Gonçalves y Elisabete Monteiro de Aguiar Pereira son las autoras del capítulo Educação geral na formação de médicos e pedagogos: contribuições para a profissionalidade. Las autoras tienen como objetivo analizar las contribuciones de la formación universitaria para una actuación como "profesional-ciudadano" (PEREIRA, 2007) para egresos de los cursos de Pedagogía y de Medicina de la Universidade Estadual de Campinas. Ellas buscan identificar aspectos de Educación General a lo largo de la formación, ya que la Unicamp ha proporcionado una flexibilización en los currículos y vivencias universitarias diferenciadas. Se parte desde el supuesto de la importancia de una formación universitaria que ultrapase los límites de la formación técnica y profesional, y que busque la formación del ciudadano crítico y ético, de pensamiento reflexivo, habilitado para una actuación con responsabilidad social (GOERGEN, 2010). Como resultados, Gonçalves y Pereira identificaron que más del 90\% de esos egresos se encuentran actuando en su área de formación y tienen autopercepción de que actúan como profesionales-ciudadanos. Según las autoras, el $70 \%$ de los egresos evalúa asimismo que esta actuación está relacionada con la formación ofrecida en la universidad, que posee elementos de Educación General que han posibilitado una formación de profesionales-ciudadanos.

En el capítulo denominado Movimentos sociais e universidade popular no Brasil, Joviles Vitorio Trevisol presenta resultados parciales de un proyecto de investigación desarrollado entre 2011 y 2013 que versó sobre la participación de atores de la sociedad civil en el proceso de creación e implantación de la Universidade Federal da Fronteira Sul. Los objetivos fueran situar a los principales actores sociales y políticos que se involucraron en el proceso; comprender el papel que los movimientos sociales ejercieron, e identificar las principales estrategias de acción movilizadas. Por medio de investigación documental y entrevistas con los principales liderazgos del Movimiento Pro-Universidad de los estados brasileños de Rio Grande do Sul, Santa Catarina y Paraná, el autor busca registrar el proceso de creación y desarrollo de la Universidad, además de reflexionar críticamente sobre sus procesos y contradicciones. Resaltó también que este proceso es fruto de una exitosa experiencia política de organización y movilización de los movimientos sociales en Brasil y América Latina, lo que cuestiona la tradición elitista de Educación Superior.

Con objeto de analizar la implantación del Plan Nacional de Formación de Profesores de la Educación Básica (PARFOR) en el estado brasileño de Mato Grosso do Sul, Margarita Victoria Rodríguez y Silvia Helena Andrade de Brito presentan una contextualización histórica sobre las políticas de formación de profesores y del escenario político y económico que anteceden al PARFOR, indicando también la legisla- 
ción nacional y estadual que reglamenta el Plan y sistematizando los datos estadísticos sobre la formación inicial de profesores en el estado. Como resultados, las autoras señalan la disonancia entre teoría y práctica en la implementación del PARFOR, considerando que la oferta de cursos no atendió a la demanda real. El PARFOR fue instituido en el estado, pero sin atender a la demanda existente, a pesar de la aceptación de parte de los profesores y de la disposición de las instituciones para ofertar los cursos de licenciatura.

En el último capítulo de la parte sobre los estudios de caso, Luiz Carlos Lückmann presenta $O$ modelo comunitário de Educação Superior, que viene siendo instituido en el Sur de Brasil, especialmente en Santa Catarina (ACAFE) y en Rio Grande do Sul (COMUNG), dando especial énfasis a las bases jurídico-legales que caracterizan este modelo y del papel político-social que ejerce en el contexto de la educación brasileña. Él aborda el tema a partir de una perspectiva histórica de esta modalidad de institución y de datos estadísticos. Para el autor, las Instituciones Comunitarias de Educación Superior (ICES) constituyen modelo de educación superior diferenciado de los demás modelos contemplados por la legislación brasileña - no público, tampoco privado -, considerándolo como exitoso y único, obteniendo éxito como medio de interiorización y democratización del acceso a la educación superior.

Dando inicio a la tercera parte del libro, Perspectivas mercadológicas na produção de conhecimento, Regina Maria Michelotto, Catarina Almeida Santos y Afrânio Mendes Catani discuten A produção do conhecimento na expansão da educação superior no Brasil con el objetivo de realizar levantamiento bibliográfico sobre la Educación Superior, desde 1996, hasta 2011, teniendo como base la revista Educación y Sociedad, publicada por el Centro de Estudios Educación y Sociedad (CEDES), periódico en el que hay un número mayor de artículos acerca del tema, en un total de veintinueve (29). Los autores verificaron que los artículos publicados por este periódico van al encuentro de las cuestiones fundamentales de la educación superior brasileña y de las políticas de educación superior, analizándolas críticamente, proclamando la fuga de tendencias hegemónicas en las discusiones científicas.

Mariluce Bittar y Wercy Rodrigues Costa Júnior analizan la política de evaluación del posgrado en Brasil, partiendo del supuesto del productivismo y del cuantitativismo de la producción científica de los docentes en el capítulo denominado Política de avaliação da pós-graduação em educação - a criação e implementação do modelo baseado na produtividade e na quantidade da produção. Los autores recurren cuestiones históricas, los Planes Nacionales de Posgrado (PNPG), y la conceptualización que los acompaña. Concluyen que existen intereses por detrás de estas características más 
objetivas de los criterios de evaluación y que lo verdaderamente importante en la evaluación - la calidad -, es un criterio que no puede fácilmente ser estimado.

En el capítulo titulado O papel da CAPES e do CNPq após a reforma do Estado brasileiro: Indução de pesquisa e da produção de conhecimento, João dos Reis Silva Júnior, Fabíola Bouth Grello Kato y Luciana Rodrigues Ferreira buscan comprender el papel de la CAPES y del CNPq ante el reposicionamiento de la universidad pública, que ha asumido nuevos papeles sociales y económicos, con especial énfasis en el posgrado y en la mercantilización del conocimiento. Haciendo una contextualización histórica y conceptual, los autores evidencian que la génesis de una política nacional de C\&T en Brasil se confunde con la creación de dos de las mayores agencias de fomento a la investigación del país: CAPES y CNPq. Analizan el trabajo docente en el posgrado ante el más reciente PNPG y cuestionan la supuesta autonomía universitaria frente a los requisitos mercadológicos que las dos instituciones reguladoras interponen a la enseñanza y a la investigación, volviendo la ciencia, la enseñanza y la investigación predominantemente tecnicistas, aceleradas y precarizadas.

En el último capítulo, António Francisco Cachapuz escribe sobre $A$ universidade na encruzilhada de paradigmas, haciendo un análisis de los últimos 25 años de políticas de educación superior y de universidad. Él divide el capítulo en cuatro partes, siendo la primera la relación entre el Estado y la Universidad (en particular la pública) en su presente orientación neoliberal; la segunda es un análisis de algunas implicaciones de los cambios por esa vía ocurridas a nivel de gobernanza y autonomía institucional de las universidades (públicas). En la tercera parte, se analizan las tendencias de la investigación y por último, aborda la integración de los sistemas de formación y de investigación a nivel de espacio europeo de enseñanza superior, y algunos de sus efectos. El autor concluye que los sistemas de educación superior en curso no son modelos cerrados y define tres principios estructurantes: el financiamiento público debe ser transparente y no puede ser disminuido; es necesario estimular la autonomía institucional; y hay que asegurar mayor democraticidad en los procesos de decisión.

Recorriendo todos estos capítulos, la obra resulta global y repleta de puntos de vista diferenciados y capacitados para un análisis crítico del universo de la Educación Superior en toda su complejidad. El mercado como regulador de la relación entre ciencia, universidad, investigación y docencia trae en sí un abismo de contradicciones y limitaciones para el desarrollo social iberoamericano. Las influencias externas en la educación de los países de orientación neoliberal y la globalización de los padrones adoptados como ideales han transformado la Educación Superior en una herramienta 
del mercado, orientada al lucro de grandes empresas, teniendo al Estado como espectador y las políticas educacionales como medios de compensación de las diferencias sociales y económicas, siendo insuficientes para dar cuenta de la desigualdad generada por el capitalismo. Son necesarias más reflexiones y resultados como los generados por los capítulos de esta obra para que la diseminación del conocimiento llegue a los intelectuales orgánicos y a los agentes políticos que puedan utilizarlos con objeto de mejorar el acceso a la Educación Superior crítica, reflexiva, justa e igualitaria.

Nota explicativa:

${ }^{1}$ Versión original en portugués. Traducción al español realizada por Hildegard Susana Jung. Máster en Educación - URI; Doctoranda en Educación - Unilasalle. 
